\title{
Sachregister zu Band 90
}

Die fett gedruckten Zahlen Bb. = Buchbe:

Ablatio retinae 90, 91, 223, 224, 227, 228, 234, 238 (Bb.), 239 (Bb.), 383 (Bb.)

und Glaskörperabhebung 49

hypotonische Iritis bei -297

infolge zystoider Makuladegene-ration no

Adamantinom der Hypophysis

366 Adiesches Symptom 372 Akkommodation 280 Alkoholamblyopie 353 Allergie,

Beeinflussung der -

durch unspezifische Reizthera-

pie 92 Amaurose 118 Aminoglaukosan 204, 205 Amylnitrit bei Glaukom 207 Amyloide

Degeneration der

Bindehaut 129 Anaemie, Biermersche, Seh-

störung bei - 373 Aneurysma carotis internae,

Augensymptome bei - 371 Angiomatosis retinae 120 Angiopathie der Netzhaut 373

- $\quad$ familiäre - bei Netzhaut-Glas-körperblutungen Jugendlicher

78

Aniridie, angeborene 104

Arachnitis optico-chiasmati-ca 357

Arecolin 205

Argyll-Robertsonsches Phä-nomen 121

Argyrose der Hornhaut und Bindehaut 226

Arsenschädigungen des Seh-nerven 352

Arteria centralis retinae, Druck der -82

- $\quad$ Astverschluß der - nach Hu-stenanfall 116

Arteria hyaloidea persistens

XI5

Asthenopie, akkommodative -

nach Schädelverletzung 297 Astigmatismus, Bestimmung des

- mit der Kobaltlampe 293 AT 10, Wirkung des - auf die

Linse bei experimenteller Teta-

nie 94 Ataxia cerebellaris, Augener-

scheinungen bei - 373 Auge, Pathologie des - bei ein-

eiigen Zwillingen 105 Augenachsenstellung, Apparat

zur Messung der $\mathbf{- 1 0 0}$

bedeuten Eigenarbeiten. sprechungen.

Augenbewegungen 279Íf. Augenhintergrund, Gefäßwerte der —- 99

- gescheckter 105Augenmuskellähmungen 284Augenspiegel, Zusatzapparatur

zum Combergschen - 101 Augentropfen, Konservierung

der - durch die Ester der p- 
Oxybenzoesäure 100 Augenverletzungen, perforie-

rende 230 - durch Preßluft 103

BakterienfloraderBindehaut327

Barth-Bildnis in der I. Wiener Universitätsaugenklinik 276

Basedowsche Krankheit, Ex-ophthalmus bei - 51

Beleuchtungsmessung bei Seh-prüftafeln 81

Bewegungsapparat des Auges 279 (Bericht)

Bindehaut s. Conjunctiva

Binnenapparat der Epithelzelle 108

Blennorrhoe, lokale Hitzeanwen-dung bei - 122

Blepharochalasis, Operation der

- $\quad$ 319Blepharoplastik 317Blepharospasmus-Brille 225Blickkrampf 287Blicklähmungen

286

Blut, Veränderungen des - bei

Glaukom 198 Blutbild bei Trachom 342 Blutdruckmessungen mit einem

neuen Dynamometer 166 Blutdruckveränderungen,

Histopathologie des Auges bei

- $85^{\circ}$

Buddsche Krankheit, Augen-

erscheinungen bei - 202 Buphthalmus 203

Carotis, Zentralarteriendruck un-ter dem Einfluß temporärer Aus-schaltung der - 82

- Augensymptome bei Aneurysma der - 371

- $\quad$ Sympathektomie der - beiGlaukom 211

Chalazion 312

Chiarische Krankheit, Augen-

erscheinungen bei - 202 Chiasma 357

- $\quad$ Erweichungsherde im - 241Chininamblyopie 13

Sachregister

zu Band 90.

385

Chlorose mit Augenerscheinungen

382 Cholesterinkristalle, Ablage-

rung von - an der Rückfläche

der Hornhaut und der Vorder-

fläche der Linse 108 Chorioidea, Blutdruck in den Ge-

fäßen der - 85 Chorioideasarkome 119

- Biologie der - 106Chorionepitheliom, Augenme-

tastase eines - beim Manne 107 Christian-Schüllersche

Krankheit 56 Cirrositas vasorum retinae 98 Conjunctiva 324 (Bericht)

- $\quad$ amyloide Degeneration der -129

- $\quad$ Argyrose der - 226Conjunctivitis tuberculosa,

Zusammentreffen einer - mit dem Aufschießen von Phlyk-tänen 1 Cornea, Argyrose der - 226

- $\quad$ Sarkom der -232

- $\quad$ Streifentrübung der - im dia-betischen Koma 112

-- Schädigungen der - durch Trä-nengas 266 
- $\quad$ Veränderungen am Nervenappa-rat der - nach Exstirpation desGanglion Gasseri 89

- $\quad$ Varizellen der - 122Cyclophorometer 102

Dakryoadenitis 321 Dermoidzysten der Lider 313 Diathermie bei akutem Glaukom

207 - Veränderungen am Auge nach

diath. Koagulation 50 Doryl 206 Dunkelanpassung, Verfahren z.

fortlaufenden Aufzeichnung der

- - ior Druck, Messungen des - an der

Zentralarterie 82,83

- $\quad$ in den Gefäßen der Chorioidea

85 Druck, intraokularer, Bezie-hungen zwischen Blutdruck und - 199

Wechselbeziehungen des intra-kraniellen und -18

Mittel zur Herabsetzung des -

95

Beeinflussung des - durch in-nersekretorische Stoffe 119

Eigenblutinjektionen bei Glaukom 207

- $\quad$ bei Trachom 343Eigenharn, Heilung eines Quin-

ckeschen Ödems und hartnäcki-

gen Ekzems durch Injektion von - 381 Einschlußkörperchen 88, 338 Ekzem, durch Injektion von

Ei-

genuringehalt geheilt 381 Elliotsche Operation 210 Enophthalmus 51 Entropium, Operation des

- 318 Enzephalitis epidemica, Au-

gensymptome bei - 365 Enzephalomyelitis, disseminier-

te nichteitrige - und multiple

Sklerose 364 Enzephalopathie, Glaukom bei

- 202

Epithelzelle, Binnenapparat der

- $\quad 108$

Erbkranker Nachwuchs, Auf-gaben des Augenarztes bei der Verhütung des $\mathbf{- 1}$-61, 64, 66

Erweichungsherde im Sehnerv, Chiasma und Tractus opticus 241

Exophthalmus 51

Farbenasthenopie 97

- $\quad$ hypoxämische 379Farbensinnprüfung bei der

Reichsbahn 125 (Bb.) Febris uveoparotidea Heer-

fordt 232 Feuerstar 42

Fissura orbitalis superior 57 Fremdkörper, Lokalisation der

- $\quad$ nach Comberg 103Frühj ahrskatarrh 330Fusion 280

Ganglion Gasseri, Veränderungen am Hornhautnervenapparat nach Exstirpation des - 89

Gehirnabszeß 233, 381

Gehirndruck 370

Gehirngeschwülste 238 (Bb.), 366 if.

Gehirnkrankheiten, traumati-sche 380

Gicht 123

Glaskörper 47 (Bericht)

Glaskörperabhebung 79, 80

- bei Glaucoma simplex 111Glaskörperblutungen, famili-

äre Angiopathie bei - 78 Glaukom 196 (Bericht) 
Glaskörperabhebung bei - Ill

und Glaskörperquellung 47 Glaukomoperationen 95, 118,

207

- $\quad$ hintere Sklerotomie 115

- $\quad$ Einfluß druckentlastender Ope-rationen auf das nichtoperierteAuge 259

Glaukosan 205

Gliom, primäres - der Retina 105

386

Sachregister

zu Band 90.

Gonokokken, Übertragbarkeit

der - auf die Conjunctiva des

Kaninchens 122 v. Graefes Briefe an Donders

125 (Bb.) v. Graefes Vorlesungen 32 Grubenbildung der Papule bei

Betrachtung mit dem gewinkel-

ten Mikroskop 104

Hemianopsie, bitemporale 357 Heterophorie 281 Histidin bei Glaukom 206 Hornhaut s. Cornea

Hypophysisgeschwülste 366,

367 - Augenhintergrund bei - 355,

356, 357- 358 Hypoxämische Farbenasthe-nopie 379

Impletol 301 Insulin und Auge 78 Iridektomie bei Glaukom 209

- $\quad$ Ablösung der Linsen-Kapsel-lamelle bei der - 119

Iridenkleisis bei Glaukom 210 Iritis, hypotonische - bei Amotio retinae 297

Kalkstoff wechsel und Auge 94 Kammerwinkelverschluß bei

Glaukom 128 Karzinome der Augenlider 315 Karzinommetastasen in Cho-

rioidea und Iris 226 - isolierte - an Iris und Ziliar-

körper 290 Katarakt, Entstehungder - 35ff. - disseminata subepithelialis 198

electric a 74

Komplikation der - durch ein akutes Glaukom 201

Sterilisation bei angeborener - 64

Kataraktoperation, Ritzmesser zur Anlegung einer Vornaht bei - 227 Keilbein und Optikus 229

Keratitis parenchymatosa 72

- $\quad$ bei Lues congenita der zweitenGeneration 146

Keratoconj unctivitis sicca 73 Keratoplastik, Fixation des

Transplantates bei der - 30õ Kleinhirntumoren 369 Kobaltlampe, Naheprobe und

Astigmatismusbestimmung mit

der - 293 Kolloidometer 102 Koma diabeticum, Streifentrü-

bung der Cornea im ·- 112 Konvergenz 280 Kurzwellenbehandlung 6, 96

Lagophthalmus, operative $\mathrm{Be}^{\wedge}$

handlung des - 231 Lamina vitrea, hyaline Degeneration der - 86 Lehrfilmind. Augenheilkunde 109 Lenticonus posterior 231 Lichtreflex der Pupille, isolierter

Ausfall der - 121 Li der 311 (Bericht) Linse 34 (Bericht) - Altern der - 73 - - Wirkung des A T 10 auf die - 94 Linsenkapsellamelle, Ablösung der - bei der Iridektomie 119 Linsentrübungen, hereditäre 63 Lymphangiom der Orbita und 
des Gesichtes 97 Lymphome der Bindehaut 334 Lymphosarkomatose der Augenlider 313

Lysocym 320, 341

Macula lutea, angeborene Degeneration der - 58, 59

- $\quad$ Verlauf der Fasern der - -358 ,

359

lochähnliche Affektion der - 295, 296

Ablatio retinae infolge zystoi-der Degeneration der - no

Magnetlanze, Magnetpfriem und

Magnetpinzette 193 Malleuserkrankung des Lides

313

Megalokornea 192

Melanosarkom, weit vorgeschrit-tenes 31

Membrana Descemeti, Ablösung der - 225

Meningiome 117, 366

Meningitis serosa 370

- $\quad$ Stauungspapille bei - $\mathbf{a}$ 354Meningoenzephalitis tuber-

culosa, Augensymptome bei -

365

Mikroskop, abgewinkeltes - zur Untersuchung des Glaskörpers und des Augenhintergrundes im Lichtbüschel der Spaltlampe 101, 104

Mitbewegung des Augapfels, ei-genartige Form der - 106

Mundschleimhaut, Transplantation der - auf die Bindehaut

334 - - Verwendung der - bei Toti-

Operation 109 Musculus obliquus superior,

Verwendungsmöglichkeit des -

als Einwärtswender 109 Myasthenie 288 •- Augenmuskulatur bei der M.

gravis pseudoparalytica 372

Sachregister

zu Band 90.

387

Naevus flammeus und Glaukom

203 Naheprobe mit der Kobaltlampe

293 Naturheilverfahren, Grenzen

des - bei Augenkrankheiten 93 Nebenhöhlenaffektionen und Augenkrankheiten 57

- Stauungspapille infolge von -

354 Nebenschilddrüseninsuffi-

zienz 93 Nervus nasociliaris 57 Nervus opticus 350 (Bericht) घ- Erweichungsherde im a- 241

- $\quad$ und Keilbein 229

Nervus opticus-atrophie 119,

355.356 - - tabische 363

- $\quad$ augendrucksenkende Behand-lung der -84

Netzhaut s. Retina Neuritis retrobulbaris 352 Nierenerkrankungen, Histopa-

thologie des Auges bei - 85 Nystagmographie 216 Nystagmus 214 (Bericht) —- vertikaler

Spontan-N. 372

- $\quad$ der Bergleute 299 (Bb.) 
Oculistica di Antonio Scarpa

383 (Bb.) Ophthalmodynamometer,

Blutdruckmessungen mit einem

neuen - 166 Optokinetischer Nystagmus

217 Or bit a 51 (Bericht) p-Oxybenzoesäure, Konservie-

rung der Augentropfen durch

die Ester der -100

Pagetsche Krankheit 56 Papille beim Kleinkind 351

Grubenbildung der - bei Be-trachtung mit dem gewinkelten Mikroskop 104

Ödem der - mit extremer Ve-nenstauung bei Stauung in der oberen Körperhälfte 120

Paratrachom 338 Parinaudsche Conjunctivitis

$33 \mathrm{i}$

Periarteriitis nodosa der Netzhaut 100 Perimeter, Projektions-P. 100 Phakomatose, eine vierte -

75 Phlyktänen, Zusammentreffen einer Conjunctivitis tuberculosa mit dem Aufschießen von - 1

Phlyktänulose 330 Pigmentdegeneration, rasch verlaufende - der Netzhaut 65

Pigmentierung, eigenartige $-\gamma-$ des Augenhintergrundes 105

Pilzerkrankungen der Binde-haut 332

Plasmome der Bindehaut 334

Preßluftverletzungen des Auges 103

Projektionsperimeter 100

Pruritus des Auges 311

Ptosis, hereditäre kongenitale 319

Pupillenabstandsmesser 101

Pupillenreflex, isolierter Ausfall des Lichtreflexes 121

Pupillenstörungen bei Hypo-physisgeschwülsten 367

Quinckesches Ödem durch In-jektion von Eigenurin geheilt 381

Reiztherapie, Beeinflussung ei-nes allergischen Zustandes durch unspezifische a- 92

Retina, Cirrositas vasorum der -98

- - Periarteriitis nodosa der $\mathbf{\square}-100$

Zysten der $\mathbf{a -} 90$

Ödem der - mit extremer Venenstauung bei Stauung in der oberen Körperhälfte 120

- - rasch verlaufende Pigmentdegeneration der - 65

- $\quad$-blutungen, familiäre Angiopa-thie bei - 78

Retinitis albuminurica, Ent-stehung der Stauungspapille bei

$\sim 354$

Retinitis diabetica 76

Retinitis pigmentosa, Versu-che therapeutischer Beeinflussung der - 157

- $\quad$ augendrucksenkende Behand-lung der $\mathbf{~ - ~} 84$

Retraktion 288 Rheinisch-Westfälische Au-

genärzte, Geschichte des Ver-

eins der - $124(\mathrm{Bb}$.) Rheumatische Gefäßentzün-

dungen 120 Rheumatismus 123 Ritzmesser zur Anlegung einer

Vornaht bei Staroperation 227 Röntgenkatarakt 43 Röntgenstrahlenschädigung

des Auges 119 Rückenmarkskrankheiten,

traumatische 380

Sarkom der Hornhaut 232 Schädelverletzung, Augenbe- 
schwerden nach - 296, 297 Schielen 281

- $\quad$ Konservative Behandlungsme-thoden bei - 291

388

Sachregister zu Band 90.

Schläfenlappentumoren, Au-gensymptome bei - $-367,368$

Schwachsichtigkeit, Gerät zur Übung des zentralen Sehens bei funktioneller - 102

Schwangerschaft, bitemporale Gesichtsfelddefekte in der - 357

Schwangerschaftsunterbre-chung 237 (Bb.)

Sehen, Gerät zur Übung des zentralen - - bei funktioneller Schwachsichtigkeit 102

Sehnerv s. Nervus opticus

Sehbahn 350 (Bericht)

Sehprüftafeln, Beleuchtungs-messung bei - $-\cdot 81$

Sehschärfe und Erwerbsfähigkeit 116

Sehschwäche, Ursachen der - 114

Ski era, hintere Ziliarnervenschlei-fen der -248

Skleritis posterior 232

Sklerose, multiple 363

- $\quad$ und Stauungspapille 355Sklerotomie, hintere - bei Glau-

kom 115 Skotom, objektiver Nachweis zen-

traler - 81 Staphyloma posticum verum

276 Starkstromkatarakt 43 Stauungspapille 353, 354, 355 Sterilisation bei angeborener Katarakt 64 Subarachnoidealblutungen

$37 \theta$ Sympathektomie der Carotis bei

Glaukom 211 Syphilis der Bindehaut 331

- $\quad$ des Nervensystems und Auge362

- ätiologische Bedeutung der - für das Glaukom 129

Tabakamblyopie 353 Tarsitis trachomatosa 341 Tarsus, tuber kulöses Geschwür

am - 113 Tetanie, Wirkung des A T 10 auf

die Linse bei experimenteller -

94

Theorien zum Selbstzweck 310

Thermo-Sklerotomie bei Glaukom 212

Toti-Operation, Verwendung der Mundschleimhaut bei $\mathbf{-}-109$

Trachom 263, 335 (Bericht)

Trachozid 343

Tractus opticus, Erweiterungs-

herde im - 241 Tränengas, Augenverletzung

durch -103

- $\quad$ Hornhautschädigungen durch -266

Tränennasengangverschluß, angeborener 273 Tränenorgane 319 (Bericht) Tränenträufeln, Pathogenese

des - 117 Traumatische Erkrankungen

des Gehirns und Rückenmarks

380 Trepanations-Zyklodialyse

212 Trichiasis, Operation der - 318 Tuberkulose des Auges 107 
der Bindehaut 330, 331

tub. Geschwür am Tarsus 113

als Berufskrankheit 229

spezifische oder naturgemäße Behandlung der - 91

Tularämie 313, 331 Turn or en der Lider 313 ff.

- $\quad$ der Conjunctiva 323Turmschädel 56

Ulcus, tuber kulöses - am Tarsus

113

Ulcus serpens fulminans in Ultrakurzwellenbehandlung

96 Unfallversicherung, Ursachen-

begriff der - 234 Unfruchtbarmachung 237 (Bb.) Ursachenbegriff der Unfallversicherung 234

Uvea, Bewertungen des Allgemein-befundes bei entzündlichen Erkrankungen der - 231

Varizellen der Hornhaut 122 Vitamin C, Entstehung des - in

der Linse 35 ff. Vorderkammer, Drucksteigerung

durch Eindringen einer Cilie in

die - 202 Vorderkammerlichtweg, Kol-

loidometer zur Ermittelung der

Helligkeitsveränderungen des -

102

Ziliarnervenschleif en, hintere

-. - der Sklera 248 Zwillinge

Pathologie des Auges

bei eineiigen - 105 Zyklodialyse bei Glaukom 209 